\title{
Autonomous Robotic Monitoring of Underground Cable Systems
}

\author{
Bing Jiang, Student Member, IEEE, Alanson P. Sample, Student Member, IEEE, \\ Ryan M. Wistort, Student Member, IEEE, and Alexander V. Mamishev, Member, IEEE
}

\begin{abstract}
Maintenance of distributed infrastructures requires periodic measurement of many physical variables at numerous locations. This task can potentially be accomplished with autonomous robotic mobile platforms. The challenges in realizing this vision include electromechanical design of the robot itself, integration of sensors able to estimate the physical properties of the infrastructure, and autonomous operation. This paper describes the electromechanical and sensing system design of the autonomous robot for the inspection of electric power cables. The multi-processor distributed control architecture developed in this project allows real-time operation of multiple sensors and a possibility to switch between an autonomous mode of operation and a remote-controlled one. The diagnostic sensor array includes thermal, visual, dielectric, and acoustic sensors for the measurement of cable status. Laboratory tests demonstrate the ability of integrated sensors to measure parameters of interest with the resolution required by the application. Field tests in the underground cable system demonstrate the ability of the designed platform to travel along the cable, navigate typical obstacles, and communicate with the host computer.
\end{abstract}

Index Terms - Autonomous monitoring, mobile robot, crawler, sensors, underground cables, aging status.

\section{INTRODUCTION}

$\mathrm{T}$ he development of autonomous robots for monitoring and maintenance of distributed infrastructures is becoming feasible for a broad range of industrial and scientific applications. The progress in this area is driven by the advancements in such enabling fields as ubiquitous computing, AI technologies, wireless communication, sensing, and power scavenging. The deployment of robots can bring such advantages over traditional monitoring and maintenance methods as lower cost, higher measurement accuracy, and greater reliability of system operation. An example of distributed infrastructure subjected to periodic maintenance tasks is the distribution level power cable system. A medium-

This project is supported primarily by the NSF CAREER Award \#0093716 to Alexander Mamishev. It is also partly supported by the Electrical Energy Industrial Consortium and Advanced Power Technologies (APT) Center at the University of Washington. The industrial members of the APT Center include AREVA, Bonneville Power Administration, CESI, LG Industrial Systems, Mitsubishi Electric, PJM, and RET.

B. Jiang, A. P. Sample, R. M. Wistort, and A. V. Mamishev are with the Sensor, Energy, and Automation Laboratory, Department of Electrical Engineering, University of Washington, Seattle, WA 98195 USA (phone: 206-221-6440, fax: 206-543-3842, e-mail: \{bjiang, alanson, rwistort, mamishev\}@ee.washington.edu). voltage distribution cable system is selected as a test-bed for the autonomous mobile platform designed for periodic measurement of system parameters.

Due to the deregulation and the resulting increasing competition among utilities, the economic efficiency of daily operations is becoming increasingly important in power industry. One of the most costly tasks in the power industry is maintenance of power system infrastructure, namely, generating plants, transmission lines, substations, and distribution networks. A large portion of electric power distribution is accomplished through cable networks. A typical power utility maintains millions of miles of installed cables. Some of these cables are direct buried (as opposed to be buried in pipe conduits) and therefore are not easily adaptable for robotic maintenance discussed in this paper. On the other hand, many urban cable installations, targeted in this project, are installed in tunnels, conduits, or pipes, which makes them accessible for inspecting robots.

Existing cable maintenance practices fall into one of the two categories: unplanned maintenance or planned maintenance. Unplanned maintenance is a response to a failure that may have caused a power outage. Planned maintenance is a scheduled inspection or replacement of power cables. Although planned maintenance ultimately delivers a more reliable continuous service, it is not an economical option for utilities. High reliability of an installed network requires conservative estimations of the remaining cable lifetime. Premature replacement of cables leads to economic losses, which could be avoided if the replacement decision were based on the specific site data rather than on generic estimates. Condition-based maintenance is often viewed as a possible solution in the industry. Case studies showed that up to $2 / 3$ of the cable systems scheduled for replacement could be kept in service with predictive diagnostics [1,2]. A key component of condition-based maintenance for cable systems is obtaining accurate information about the condition of each cable.

Existing techniques for monitoring the aging of distribution networks require manual inspection of individual cables by maintenance staff or by outside consultants. The instrumentation used for such tasks varies from simple handheld devices to vans equipped with highly sensitive measurement devices $[3,4]$. In all cases, the cable inspection is a costly process. A broad spectrum of sensing principles is used for the inspection tasks. Some of these sensing methods, especially acoustic detection, are greatly enhanced by the ability to take measurements along the cable, as opposed to 
relying on measuring parameters at the ends of the cables.

The goal of this project is to develop an autonomous robotic platform that can inspect underground power distribution cables, thus providing utilities with accurate information regularly and at a lower cost. Prior work in this field could be loosely separated in two categories: a) use of robots for power system maintenance tasks, and b) design of crawling platforms for a variety of different applications. The following two paragraphs provide a brief overview of each category, respectively.

Several types of autonomous and semi-autonomous robots have been developed for maintenance of power systems over the last two decades. In 1989, Tokyo Electric Power developed two robots that traversed and monitored fiber-optic overhead transmission wires [5]. These robots were equipped with a remotely operated stereoscopic TV camera system designed to facilitate maintenance of energized high voltage power lines. The method has not achieved widespread use so far. Another project that took place in Japan, in 1991, was an autonomous mobile robot that inspected the power distribution system of railroads [6]. A segmented body design was useful for implementing obstacle avoidance techniques. Although not widely used either, this system has been reported to produce positive results. A compact tele-operated trolley for live-line operations was developed at HydroQuebec's Research Institute in 2000, intended primarily for de-icing of transmission line conductors [7]. Robots were also developed for the monitoring of the nuclear power plants $[8,9]$.

There are many examples of semi-autonomous robots and crawlers used for inspection and maintenance purposes of distributed systems. Pipe crawling inspection robots are commonly used for leakage inspection of oil and gas pipes [10-12]. Pipe crawler applications also include rescue and explosive detection [13]. None of the previous projects address underground power cables. Many different driving mechanisms and control algorithms are adopted for internal pipe crawlers [14-16], however, they are not directly applicable to power cable environment. The operating environment, discussed in the next section, is an open space, which has more obstacles than operating the environment of pipe crawlers.

\section{OPERATIONAL ENVIRONMENT}

Since the goal of this project is to demonstrate the feasibility of the mobile monitoring of cable systems, design of a reliable autonomous robotic platform is crucial. The main requirements for the platform are: the ability to operate over large distances, operate within the physical constraints of the cable environment, avoid obstacles within the environment, and carry the instruments needed for cable inspection.

The challenges of robot design in this case are significantly different from those for the pipe crawling robots. The underground cable environment is not as geometrically simple as a pipe and requires a much more adaptable design. Fig. 1 shows an example of the cables and their surroundings in a 14 $\mathrm{kV}$ underground installation at the University of Washington.
The cables lie in metal troughs that are fairly uniform in width and height, approximately 25 to $18 \mathrm{~cm}$ wide and usually $5 \mathrm{~cm}$ deep. However, the cables themselves may be positioned anywhere in the trough: they can be in the middle, or touch the trough walls. In the latter case, a "hugging" chassis design expected of cable crawlers is not suitable. Instead, the robot needs to be above the cable while maintaining a close contact between the sensors and the cable surface. In addition, the troughs are often stacked one on top of another, which limits the amount of space available for the robot to about $11 \mathrm{~cm}$, in this particular case. There are no sharp turns because the minimum radius of curvature of a cable when bent is on the order of meters. Additionally, minor obstructions, such as splices and mounting brackets, may be encountered by the robot when traveling along the cable, and must be negotiated. Even though the overall height is limited, the space available along the length of the cable for payloads, such as sensors, is fairy flexible because several segments can be added to the robot base.

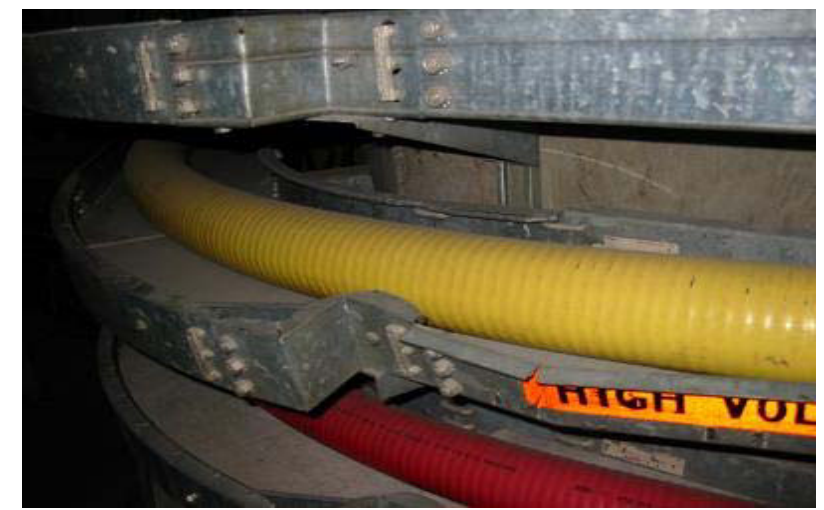

Fig. 1. A typical installation of a power cable in a tunnel at the University of Washington.

\section{Mechanical Design of Robot Base}

A modular multi-segmented design has been selected over a single platform option because it provides improved turning ability, stability when turning, and interchangeable subsections. Additionally, a multi-segmented design allows the robot to carry additional sensors. One can think of the robot as a train of subsections crawling along the cable, with different cars being interchanged for different diagnostic or service tasks. Fig. 2 shows a conceptual drawing of the present robot configuration. The platform has two segments, with one devoted to robot control and the other to sensors and data processing.

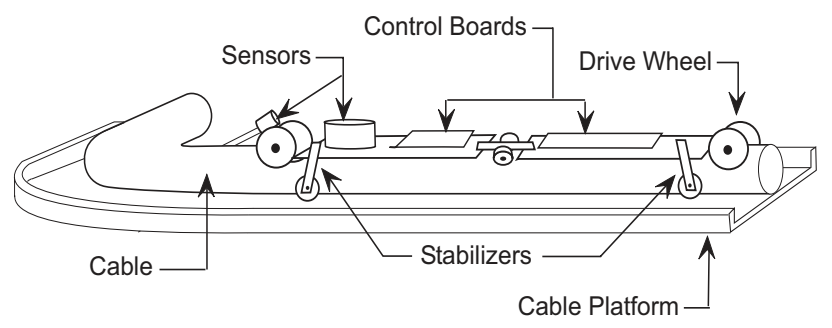

Fig. 2. Schematic of major components of the mobile monitoring platform. 
The drive sections are self-contained units that pivot freely from the base to provide effective turning. Propulsion is provided by two 12-volt DC gearhead motors, which drive hourglass-shaped neoprene wheels located at both ends of the robot. Optical encoders are attached to the drive shaft of each wheel for tracking speed and measuring distance traveled.

Each segment of the robot base contains a set of stabilizers, which keep the robot platform on the cable. The stabilizers have the ability to retract, in order to negotiate obstacles such as a splice in the cable or a mounting bracket.

Fig. 3 shows the robot on a piece of test cable and trough. In the foreground is the front drive section followed by a set of stabilizers and a section for carrying sensors.

Since the robot is intended as a mobile monitoring platform, sensing requirements play a central role in the design of the sensor-carrying platform. While many of the sensors do not require contact with the cable, the acoustic emission sensor must have proper contact with the cable surface in order to make accurate measurements. A balanced belt-driven mechanism is used to drive the sensor up and down for periodic measurements, for which the robot has to make stops. A force feedback control loop is employed to ensure that proper contact is made, since small force cannot guarantee adequate contact while excessive force may damage the driving mechanism.

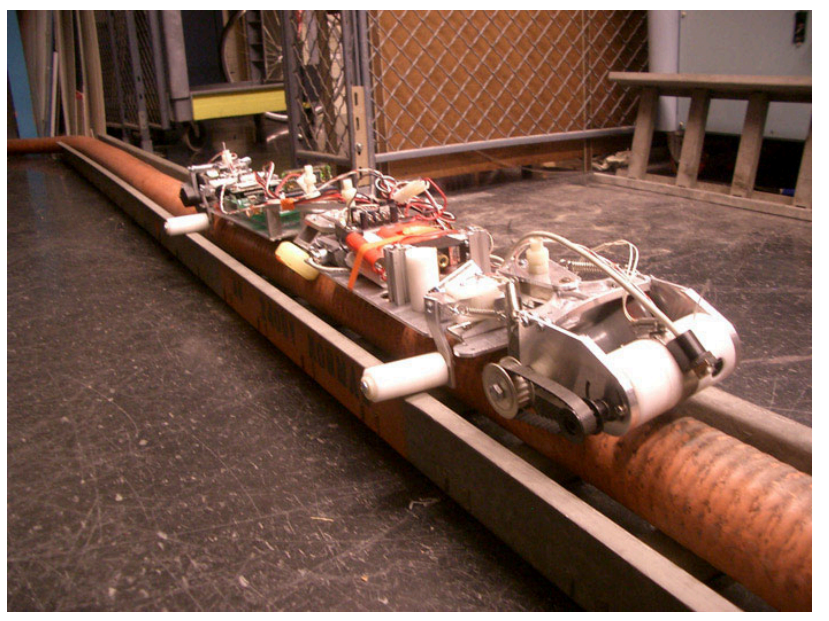

Fig. 3. Photograph of the robotic platform for the mobile monitoring of underground power cables.

\section{CONTROL}

The requirements for the control system include the need for real-time autonomous control, signal processing, data logging, and communications with an external computer. To accomplish these tasks, a distributed multi-processor architecture has been implemented. Each processor performs a specific task, and cooperates with other processors.

\section{A. Overview of Control System and Processor Architecture}

The control system is composed of three distributed units: a remote host computer, a data acquisition board, and a general control board. The host computer provides a user interface, which gives the operator the ability to view the sensor output remotely, to post-process data, and to control the robot in nonautonomous mode. All communication with the remote user interface and the robot is currently achieved through the use of $915 \mathrm{MHz}$ wireless communications modules. This communication method requires relative proximity of the robot and the host computer (tens of meters). Since the robot is expected to operate autonomously in remote tunnels, the long-range communication is not necessary. In the future, it may be possible to communicate by using the cable itself as a signal carrier, but at this stage such an increase in design complexity was deemed unnecessary. The control board provides the wireless communication device interface. It also communicates with the signal processor, thus linking all distributed processes.

\section{B. Control Board}

The control board combines all elements of robot hardware by handling wireless communication with the user interface, sensor data acquisition and logging, as well as control of the autonomous functions of the robot.

Fig. 4 shows that these tasks are distributed among four processors to increase implementation flexibility. This approach allows each task to be handled by the processor most suited for the task. One primary controller, or the host processor, handles the most important control decisions, such as navigational or motor control routines. The slave (client) processors handle smaller tasks, for example obtaining sensor data and handling low-level actuator control signals. Interprocessor communication within the board is accomplished through use of the serial peripheral interface (SPI) bus and is initiated by interrupts creating bilateral communication between processors.

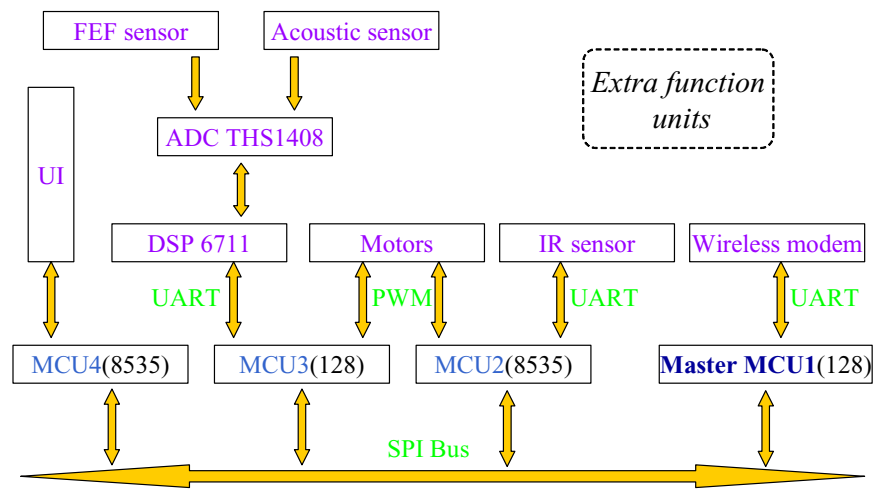

Fig. 4. Diagram of the robotic platform's control board.

Fig. 5 shows a wireless communications protocol developed to increase data transfer reliability between the control board and the user interface. Likewise, a protocol has been developed to handle communication between processors within the control board. Communication protocols have also been designed for data transfer between the control board and the digital signal processor, which is also interfaced with serial communications.

\begin{tabular}{l|l|l|l|l|l|l|l|l|l|}
\cline { 2 - 7 } Host & CR & CMD & CRC & CR & \multicolumn{6}{|c|}{} \\
\cline { 2 - 8 } & & & & & Execute & CR & Data & CRC & CR \\
\hline
\end{tabular}

Fig. 5. Communication protocol for wireless communication, where $\mathrm{CR}$ means a Carriage Return, CMD means a command, and CRC means a cyclic redundancy check. 


\section{Data Acquisition System}

Data is transferred through a serial communication link to the control board from dielectric, acoustic, thermal, and video sensors. Diagnosing failure from dielectric and acoustic data requires high speed signal processing. By processing acoustic data locally on the robot, the task of storing large amounts of data is eliminated and autonomous measurement mode becomes more effective. This allows for greater flexibility of selecting a measurement protocol when multiple readings are required to achieve accuracy. A digital signal processor (DSP) board has been implemented to obtain high frequency acoustic data, process the data, and return the processed results to the control board. Using a DSP system is ideal for this application. The use of a DSP also gives the potential to decrease the physical size and power consumption of the signal processor due to the scalability of the DSP architecture. An analog to digital converter is used to capture acoustic signals at the frequencies over $100 \mathrm{kHz}$. One kilobyte of data is transferred from the analog to digital converter to the DSP memory where the signal is then processed by the DSP. Multiple processed packets of data are often needed to identify a fault in the system. Once a conclusion has been generated by the DSP system, the result can be sent to the control board, to be stored or used to make decisions, which can affect the operation of the robot and sensing algorithms.

\section{Testing OF The Robot Platform}

The presented robot platform is designed to operate within the power distribution system at the University of Washington. Initial tests of the robot took place on power cables within a laboratory setting and subsequent field tests have been performed on the cable distribution system. The next research step is to send the robot out for extended periods of time, possibly days, and have it autonomously collect data from miles of cable. At this point, robot design and measurements in a supervised semi-autonomous mode are being reported.

Deployment of the robotic platform in the field consists of placing the robot on the cables and initializing it from a laptop. The graphical user interface at the host computer provides flexibility for robot operation, but is not necessary for simplified autonomous operation.

Field tests demonstrated that the platform works properly within the size constraints of the narrow trough. The robot was able to negotiate small obstacles, namely, splices, protruding bolts, and low crossbeams that support upper toughs. The robot was tested in manual and in autonomous modes, with no noticeable difference in mechanical performance. In the manual mode, the robot was able to transmit real-time signals from the onboard video camera and the thermal sensor, although the range was limited since the tests took place underground and the tunnel has turns. The autonomous mode, which allows robot operation without control from the host computer, also passed the test. The robot could maintain balance and contact with the cable at the entire range of speeds, from 5 to $20 \mathrm{~m} / \mathrm{min}$.

Energy harvesting is not currently implemented. The battery life is currently limited to about one hour of continuous movement with all sensors and wireless communication active. The future design enhancement will provide recharging and energy harvesting. The ruggedness of the robot in field conditions requires enhancement. The dripping water and high temperatures at some parts of the tunnel were avoided at this stage. The platform is designed for horizontal cables only; this is acceptable for the project since more than $90 \%$ of cables are laid within well-defined, obstacle-free horizontally troughs. Laboratory tests focused on sensors and are discussed separately in the next section.

\section{Sensor Selection, Design, AND Testing}

This mobile monitoring system utilizes several different sensors in order to obtain information about aging of cables. Sensors have been chosen to meet the following criteria: a) sensors must be small in size and lightweight, thus allowing them to be easily incorporated into the robot platform; b) sensors must measure physical variables that help with evaluation of cable status; c) frequency of data output should be below $10 \mathrm{MHz}$, to meet the limitation of the data acquisition system; d) sensor must be sufficiently rugged to withstand mechanical and environmental impact of the operating environment. The following sub-sections provide a discussion on sensor selection and present representative experimental data.

\section{A. Infrared Sensors for Hot Spots}

Excessive heat build-up contributes significantly to the premature breakdown of the insulation in distribution cable networks. The consequences of premature cable failure include costly replacement and unexpected loss of power service. Factors that cause overheating include current overloading, physical damage, insulation aging, partial discharges, changes in ambient temperature, and proximity to other cables and water/steam pipes [17].

Overheating rarely affects the entire length of the cable. Typically, so-called "hot spots" form at the points of excessive mechanical stress, water seepage, or crossings with other cables. The industrial solutions for detection of hot spots include installing fiber optic sensors along the cable length, manual inspection, or avoiding the problem and waiting until the cable fails (which is acceptable in some situations). The method presented here has never been tried with power cables and still has to prove its usefulness in an industrial setting.

Infrared sensors do not need physical contact with the cable. Therefore, they can continuously take temperature readings as the robot travels along the cable, without the need to stop to take intermittent measurements. In a few years, as technology develops, infrared sensors are likely to be replaced with infrared cameras in this application. Temperature data is acquired continuously and used for used by the control board for detection of possible incipient faults. An increase in temperature from $75^{\circ} \mathrm{C}$ to $90^{\circ} \mathrm{C}$ causes an increase in aging by a factor of two in thermoplastic polyethylene, and an increase by a factor of 3.7 in cross-linked polyethylene [18]. In addition, unfavorable conditions in the surrounding 
environment, such as cables crossing under a paved street or parking lot, may result in temperature rise of $10^{\circ} \mathrm{C}$ to $20^{\circ} \mathrm{C}$ [19]. Fig. 6 shows an artificially created hot spot measured by the infrared sensor $\left(\right.$ Raytek $^{\circledR}$ MID) in the laboratory conditions. The measurement was made in an autonomous mode as the robot was driving along the cable. The trigger condition in this case was the $15^{\circ} \mathrm{C}$ increase above the mean ambient temperature. As experiments continue, much more sophisticated pattern recognition algorithms will be used for pre-processing of sensor data.

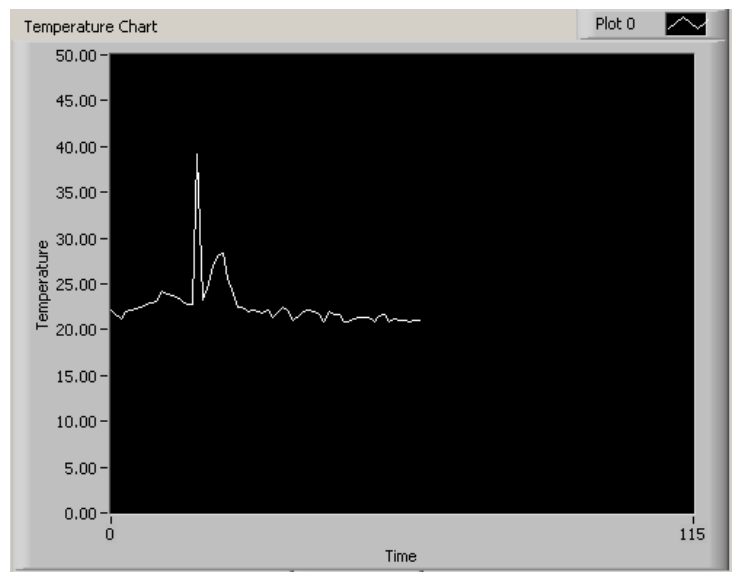

Fig. 6. A screen snapshot of the temperature measurement time sequence in the user interface. This sequence is stamped for being recorded in memory for download to the host computer. When the hot spots are not detected, the data is discarded from the memory.

\section{B. Sensors for Partial Discharges}

Partial discharges (PDs) occur when a failure in the cable's insulation causes a rapid energy release, usually resulting in a detectable acoustic vibration. In many cases, PDs are a precursor to total cable failure, and therefore are an important tool for estimating the remaining service life of a power cable.

Attenuation of the acoustic wave makes it impractical for conventional "static" distributed sensor methods to use this monitoring technique. However, since the robot platform is mobile, it can be positioned within a detectable range (approximately a meter), making acoustic sensing possible. There are significant advantages for using acoustic sensing. For instance, an acoustic emission sensor is not affected by electrical interference.

Acoustic sensing has been very successful for switchgear and transformers [20]. However, the accurate recognition of the acoustic emissions from a partial discharge in the overall vibration pattern of the cable is a challenging task. Attenuation pattern and the signal time-frequency characteristics are both important factors in identifying a PD. These characteristics are both dependent on the internal geometry of the cable, interfaces between the materials, absorption by the material (higher frequency components are removed), and the frequency-dependent propagation.

One of the most significant challenges when implementing the acoustic emission sensor is processing large amounts of data, which requires considerable computational resources. This is problematic due to size constraints and the harsh operating environment found in underground networks. However, the ability to transmit the raw data back to the host computer via wireless connection is not always guaranteed. Therefore, the processing of the acoustic emissions data will take place on board and the results can then be relayed back to the host computer when a connection is available.

\section{Dielectrometry Sensors for Aging Status}

Measurement of dielectric properties of the outer layer of cable insulation can provide valuable insights into its status. The most obvious application is the measurement of presence of water in the bulk of the cable insulation. Water trees and electrical trees are the most common causes of partial discharges. These trees typically develop from a small initial crack, void, or delaminated area. It takes several weeks to several years before the incipient fault causes cable failure. The dielectric spectroscopy sensor is installed on the robot to make continuous measurement of the cable surface and detect abnormalities, which are not necessarily at the surface of the insulation layer. The periodic measurement, admittedly, does not guarantee detection of all processes. Partial discharges may be intermittent in time, as environment conditions change. For example, a cable with a small crack may remain dry and be in working condition in the dry hot summer. As weather changes, water may act as a conductor after this crack becomes wet, allowing for electrical discharges. In this case the acoustic sensor would not detect the water tree unless the robot was in the right place and the right time. The fringing field dielectrometry sensor may still detect the voids in the insulation, even if they are not filled with water, although the signal change would be weaker in this case. In other words, the fringing electric field dielectrometry sensor tests the actual condition of insulating material rather than the resulting partial discharge activity [21].

Measurement of dielectric properties can also determine the aging status of the insulation material. The changes of various physical and chemical properties of materials are reflected in the change of dielectric properties. However, this type of measurement requires highly sensitive instrumentation. It will be a very challenging task to use the existing sensor technology in order to obtain information about dielectric property changes in field conditions.

The shape of the dielectrometry sensor head can vary depending on the task. Usually, the sensor head looks like an array of coplanar electrodes, possibly following the curvature of the cable. The fringing electric fields emanate from these electrodes and penetrate the material under test, in our case, power cable. In the presented robotic platform design, the sensor head is periodically lowered from the robot platform on linear actuators to inspect the distribution cable.

A detailed overview of fringing electric field sensors is available in [22]. Practically all prior measurements of material properties using this method were previously accomplished using benchtop instrumentation. The introduction of E-field sensor IC chip MC33794 makes it feasible to put a FEF sensor on the robot platform. Fig. 7 shows the schematics of the FEF sensor system, which is capable of broadband frequency and multi-channel sensing. The external to the chip circuitry fits onto a printed circuit 
board with the area of about two square inches.

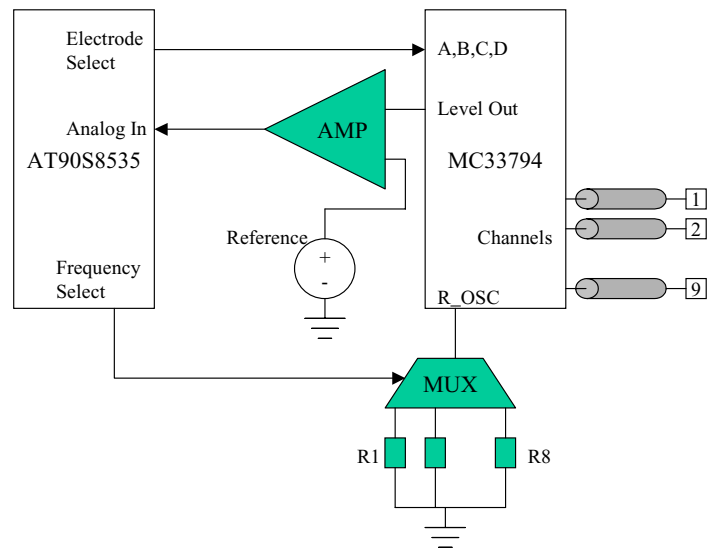

Fig. 7. Circuit diagram of the dielectric spectroscopy sensor implemented with the Motorola chip MC 33794.

\section{Video Camera}

Fig. 8 shows the image from the onboard video camera, a simple and useful additional tool for cable inspection. Coupled with a wireless user interface, an operator viewing real-time video can perform a visual inspection of the cable that may not be safely or easily accessed by maintenance personnel for example in a nuclear power plant environment. The operator can also aid in advanced obstacle avoidance and remotely troubleshoot problems, thereby enhancing the capabilities of the robot.

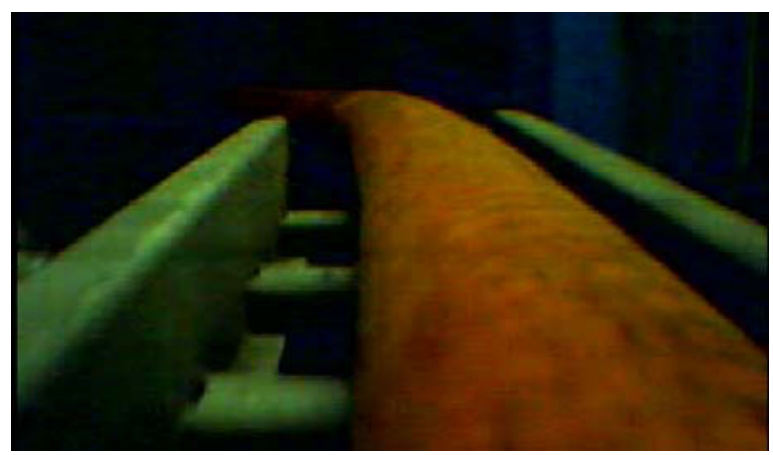

Fig. 8. View from the on-board camera transmitted wirelessly to the host computer.

\section{CONCLUSIONS}

A mobile autonomous robot for detection of incipient faults in electric power cables has been developed and tested in laboratory and in field conditions. Mechanical design and autonomous control system of the robot are optimized for the requirements of the operating environment, namely, underground tunnels of distribution power systems. The analysis of detectable physical phenomena led to a selection of necessary sensing principles. Four types of sensors for measurement of physical properties of the distributed infrastructure were integrated into the mobile platform and tested in laboratory conditions. The presented mobile inspection method provides a viable solution to the task of monitoring and maintaining underground cable systems.

\section{FUTURE WORK}

The next immediate step of this research project is to test performance of the sensors and the platform itself in a wide variety of field conditions. Ultimately, the robot platform will have to work in many different environments with many different cable configurations. Therefore, the miniaturization and the improvement of mechanical mobility of the robot are important areas of further research. The use of multiple platforms, in the framework of mobile sensor networks, is also an important direction of future work.

\section{ACKNOWLEDGEMENT}

The research of the undergraduate students has been supported by the University of Washington Mary Gates Foundation and the Electric Energy Industrial Consortium. The authors also gratefully acknowledge the invaluable assistance of Mark Kirschenbaum of the University of Washington Power Plant with experiments.

\section{REFERENCES}

[1] W. Reder and D. Flaten, "Reliability Centered Maintenance for Distribution Underground Systems," IEEE Power Engineering Society Summer Meeting, vol. 1, 2000, pp. 551-556.

[2] P. Birkner, "Field Experience With a Condition-Based Maintenance Program of 20-KV XLPE Distribution System Using IRC-Analysis," IEEE Transactions on Power Delivery, vol. 19, no. 1, pp. 3-8, 2004.

[3] E. David, N. Amyot, and J.-F. Drapeau, "Diagnostic of Field Aged Cables and Accessories by Time-Domain Dielectric Spectroscopy," 2003 Annual Report on Conference on Electrical Insulation and Dielectric Phenomena, 2003, pp. 165-170.

[4] N. H. Ahmed and N. N. Srinivas, "On-Line Partial Discharge Detection in Cables," IEEE Transactions on Dielectrics and Electrical Insulation, vol. 5, no. 2, pp. 181-188, 1998.

[5] S. Nio and Y. Maruyama, "Remote-Operated Robotic System for Live-Line Maintenance Work," ESMO-93 Sixth International Conference on Transmission and Distribution Construction and Live Line Maintenance, 1993, pp. 425-435.

[6] H. Kobayashi, H. Nakamura, and T. Shimada, "An Inspection Robot For Feeder Cables-Basic Structure And Control," International Conference on Industrial Electronics, Control and Instrumentation, vol. 2, 1991, pp. 992-995.

[7] S. Montambault, J. Cote, and M. St.Louis, "Preliminary Results on the Development of a Teleoperated Compact Trolley for Live-Line Working," Proceedings of 2000 IEEE ESMO - IEEE 9th International Conference on Transmission and Distribution Construction, Operation and Live-Line Maintenance, 2000, pp. 21-27.

[8] N.Ishikawa and K.Suzuki, "Development of a Human and Robot Collaborative System for Inspecting Patrol of Nuclear Power Plants," Proceedings of the 6th IEEE International Workshop on Robot and Human Communication, 1997, pp. 118-123.

[9] Seungho Kim, Seung Ho Jung, and Chang Hoi Kim, "Preventive Maintenance and Remote Inspection of Nuclear Power Plants Using Tele-Robotics," Proceedings of IEEE/RSJ International Conference on Intelligent Robots and Systems, vol. 1, 1999, pp. 603-608.

[10] A. Zagler and F. Pfeiffer, "'MORITZ" a Pipe Crawler for Tube Junctions," Proceedings of IEEE International Conference on Robotics and Automation, vol. 3, 2003, pp. 2954-2959.

[11] S. G. Roh, S. M. Ryew, J. H. Yang, and H. R. Choi, "Actively Steerable in-Pipe Inspection Robots for Underground Urban Gas Pipelines," Proceedings 2001 ICRA IEEE International Conference on Robotics and Automation, vol. 1, 2001, pp. 761-766.

[12] H. T. Roman, B. A. Pellegrino, and W. R. Sigrist, "Pipe Crawling Inspection Robots: an Overview," IEEE Transactions on Energy Conversion, vol. 8, no. 3, pp. 576-583, 1993. 
[13] J. Weston, "A Safer Way to Search Disaster Sites," IEEE Robotics \& Automation Magazine, vol. 7, no. 3, pp. 56-57, 2004.

[14] A. M. Bertetto and M. Ruggiu, "In-Pipe Inch-Worm Pneumatic Flexible Robot," Proceedings of 2001 IEEE/ASME International Conference on Advanced Intelligent Mechatronics, vol. 2, 2001, pp. 1226-1231.

[15] J. A. Galvez, P. Gonzalez de Santos, and F. Pfeiffer, "Intrinsic Tactile Sensing for the Optimization of Force Distribution in a Pipe Crawling Robot," IEEE/ASME Transactions on Mechtronics, vol. 6, no. 1, 2635, 2001.

[16] S. Guo, Y. Sasaki, and T. Fukuda, "A New Kind of Microrobot in Pipe Using Driving Fin," Proceedings of 2003 IEEE/ASME International Conference on Advanced Intelligent Mechatronics, vol. 2, 2003, pp. 697-702.

[17] L. Lamarre, D. Fournier, and R. Morin, "Early Detection of Faults in Underground Distribution Cable Joints by Partial Discharge Measurements," Proceedings of the 4th International Conference on Properties and Applications of Dielectric Materials, 1994, pp. 864867.

[18] R. Lyle, "Effect of Testing Parameters on The Outcome of The Accelerated Cable Life Test," IEEE Transactions on Power Delivery, vol. 3, no. 2, pp. 434-439, 1988.

[19] H. Brakelmann and G. Anders, "Ampacity Reduction Factors For Cables Crossing Thermally Unfavorable Regions," IEEE Transactions on Power Delivery, vol. 16, no. 4, pp. 444-448, 2001.

[20] Y. Lu, X. Tan, and X. Hu, "PD Detection and Localisation by Acoustic Measurements in an Oil-Filled Transformer," IEE Proceedings-Science, Measurement and Technology, vol. 147, no. 2, pp. 81-85, 2002.

[21] A. V. Mamishev, Y. Du, B. C. Lesieutre, and M. Zahn, "Development and Applications of Fringing Electric Field Dielectrometry Sensors and Parameter Estimation Algorithms," Journal of Electrostatics, vol. 46, no. 2-3, pp. 109-123, 1999.

[22] A. V. Mamishev, K. Sundara-Rajan, F. Yang, Y. Q. Du, and M. Zahn, "Interdigital Sensors and Transducers," Proceedings of the IEEE, vol. 92, no. 5, pp. 808-845, May 2004. 